mononuclear cells (PBMCs), and a relationship among specific inflammatory mediators, ECS, and organ involvement has not been established

Objectives: To analyze ECS elements and related inflammatory molecules in PBMCs of SSc patients, and evaluate their relationship with the clinical profile of the disease

Methods: 24 SSc patients [including 5 Pre-SSc, 13 limited cutaneous SSc (Ic-SSC) and 6 difusse cutaneous SSc (dc-SSc)] and 24 healthy donors (HD) were included. Purified PBMCs were used for analysis of gene expression of molecules belonging to the ECS: CB1, CB2, GPR55, PPAR $\gamma$, FAAH, MAGL and TRPV. Inflammatory mediators were evaluated in PBMCs by RT-PCR. Clinical evaluation of patients was performed and correlation/association studies were developed

Results: Cannabinoid type-2 receptor, GPR55 and TRPV1 gene expression were reduced in PBMCs of SSc patients, while FAAH levels were elevated. CB2 levels were lower in Ic-SSc in relation to Sc-SSc, and related to the presence of auto-antibodies anti-Scl70. Concomitantly, levels of FAAH were higher in dc-SSc in relation to Ic-SSc, and associated to the presence of anti-centromere antibodies. A relationship between the low levels of GPR55 and the presence of pulmonary arterial hypertension was further demonstrated. Reduced levels of TRPV were associated to pulmonary involvement.

Analysis of the inflammatory profile showed significantly increased PBMC expression levels of IL-1, IL-8, IL-12, MCP-1, TGF $\beta$, TNF $\alpha$, and VEGF-A in SSc. In addition, in the patient's group of Pre-SSc, specific elevation in various cytokines was demonstrated (i.e.IL-1,IL-17,VEGF-A), suggesting that these cytokines might act as early biomarkers of disease development. Patient's positive for anticentromere antibodies showed increased expression of IL-4, IL-17 and MCP-1 in relation to those positive for anti-scl70. PBMCs expression levels of TGF $\beta$, IL-12 and MCP-1 were higher in Ic-SSc compared to ds-SSc. Interestingly, we observed a direct relationship between levels of these three cytokines and the occurrence of pulmonary hypertension, a pathology more frequent in Ic-SSc, thus suggesting a role for these inflammatory molecules in pulmonary involvement in this form of the disease. Correlation studies demonstrated an interrelation among deregulated expression of various molecules belonging to the ECS (i.e. FAAH, GPR55 and TRPV1) and inflammatory mediators over-expressed in serum and immune cells (i.e. CRP, ESR, MCP-1, TNF $\alpha$ and VEGF-A)

Conclusions: SSc patients show altered gene profile of ECS and inflammatory mediators in PBMCs, which might allow the discrimination between limited and diffuse forms of the disease, and are associated with the presence of specific auto-antibodies and the internal organ involvement. Our overall data suggest an appealing potential target of ECS for treatment of SSc, as it seems to be related to the inflammatory profile

Acknowledgements: CTS-794 and ISCIII (PI15/01333 and RIER RD16/0012/ 0015

Disclosure of Interest: None declared

DOI: 10.1136/annrheumdis-2017-eular.4946

\section{FRI0361 INTERLEUKIN-4 INDUCES CLASS-SWITCHING TO IGG4 AND SYNERGISTICALLY CONTRIBUTES TO PLASMABLASTS DIFFERENTIATION WITH INTERLEUKIN-21 THROUGH CD40 DEPENDENT MANNER IN IGG4-RELATED DISEASE}

M. Akiyama, H. Yasuoka, K. Yoshimoto, T. Takeuchi. Division of Rheumatology, Department of Internal Medicine, Keio University School of Medicine, Tokyo, Japan

Background: IgG4-related disease (IgG4-RD) is a lymphoproliferative disorder characterized by elevated serum levels of $\lg 44$ and increased numbers of circulating plasmablast. We have previously reported that class-switching to IgG4 and plasmablast differentiation are mediated by follicular helper type $2 \mathrm{~T}$ cells which are known to secrete interleukin (IL)-4, IL-13, IL-10 and IL-21 (1, 2). However, the cytokines which play a role in the IgG4 class-switching and plasmablast differentiation through cell to cell contact remain unclear in IgG4-RD. Objectives: The aim of this study was to elucidate the role of follicular helper type $2 \mathrm{~T}$ cell cytokines (IL-4, IL-13, IL-10, and IL-21) and cell to cell interaction in the pathogenesis of IgG4-RD.

Methods: Peripheral blood mononuclear cells (PBMCs) were prepared from seven consecutive patients with active, untreated, newly diagnosed IgG4-RD and five healthy controls. To identify the cytokines which induce IgG4 classswitching, the cells were stimulated with IL-4, IL-13 or the combination with other cytokines, such as IL-10 or IL-21. The amounts of IgG4 and IgG in the culture supernatants were measured by cytometric bead arrays. The expression level of activation-induced cytidine deaminase (AID; an enzyme essential for class-switch recombination) was analyzed by quantitative PCR to confirm the induction of class-switching by stimulation with cytokines. The numbers of plasmablasts and plasma cells induced by cytokines stimulation were examined by flow cytometry. Moreover, an anti-CD40 antibody was added to the culture to elucidate the effects of cell to cell interaction on the differentiation of plasmablasts or plasma cells. Results: IL-4 significantly induced CD40-stimulated PBMCs to undergo lgG4 class-switching in patients with IgG4-RD, while IL-13 did not show any positive effects. Moreover, the $\lg \mathrm{G} / \mathrm{lgG}$ ratio in culture supernatants was also significantly higher in the stimulation with IL-4 compared to other cytokines in IgG4-RD. In addition, the expression levels of AID mRNA were increased by stimulation with IL-4 compared to that by no stimulation or CD40 stimulation in IgG4-RD. On the other hand, PBMCs from healthy controls showed no significant difference in IgG4 production after stimulation with either IL-4 or IL-13. Furthermore, IgG4 production stimulated with IL-4 was significantly higher in IgG4-RD than that in healthy controls. Assessing additional effects of IL-10 or IL-21 on IL-4, IL-10 and IL-21 did not increase IgG4 production and IgG4/IgG ratio compared to IL-4 alone in IgG4-RD. However, importantly, IL-21 synergistically induced plasmablasts or plasma cells differentiation in combination with IL-4, whereas no obvious change was observed in PBMCs stimulated with IL-4 alone in IgG4-RD. Of note, the differentiation of plasmablasts and plasma cells by IL-4 and IL-21 was markedly abolished in the absence of CD40 stimulation.

Conclusions: Our results strongly suggest that IL-4 plays a pivotal role in IgG4 class-switching, and the effective collaboration between IL-4 and IL-21 contributes to plasmablasts and plasma cells differentiation via CD40 dependent manner in patients with IgG4-RD.

References:

[1] Akiyama M, et al. Arthritis Res Ther. 2016;18:167.

[2] Akiyama M, et al. Arthritis Rheumatol. 2015;67:2476-81.

Acknowledgements: We thank all the physicians and others caring for the patients enrolled in our study.

Disclosure of Interest: None declared

DOI: 10.1136/annrheumdis-2017-eular.3040

\section{FRI0362 ANGIOGENIC T CELL EXPANSION CORRELATES WITH SEVERITY OF PERIPHERAL VASCULAR DAMAGE IN SYSTEMIC SCLEROSIS}

M. Manetti, S. Pratesi, E. Romano, I. Rosa, A. Martiradonna,

S. Bellando-Randone, S. Guiducci, L. Ibba-Manneschi, E. Maggi,

M. Matucci-Cerinic. Experimental and Clinical Medicine, University of Florence, Florence, Italy

Background: The mechanisms underlying endothelial cell injury and defective vascular repair in systemic sclerosis (SSc) remain unclear. Recent studies suggest that a novel T cell subset, the so-called angiogenic T (Tang) cells, may have an important impact on the repair of damaged endothelium. Tang cells are characterised by the co-expression of CD3, CD31 (platelet-endothelial cell adhesion molecule-1) and CXCR4 (or CD184, receptor for the CXC chemokine stromal cell-derived factor-1 (SDF-1)/CXCL12). Tang cells may promote the formation of new blood vessels and endothelial repair by stimulating the function and differentiation of endothelial progenitor cells possibly through the secretion of proangiogenic cytokines, thus fostering postnatal vasculogenesis.

Objectives: This study aimed to analyse the Tang cell population in relation to disease-related peripheral vascular features in SSc patients.

Methods: Tang cells $\left(\mathrm{CD} 3^{+} \mathrm{CD} 31^{+} \mathrm{CXCR} 4^{+}\right)$were quantified by flow cytometry in peripheral blood samples from 39 patients with SSc and 18 matched healthy controls $(\mathrm{HC})$. $\mathrm{CD} 3^{+} \mathrm{CD} 31^{+} \mathrm{CXCR} 4^{+}$Tang cells were expressed as a percentage of total $\mathrm{CD}^{+}{ }^{+} \mathrm{T}$ cells. Circulating levels of SDF-1 $\alpha$ were assessed in paired serum samples by immunoassay. Skin sections from patients with early diffuse cutaneous SSc $(n=7)$ and $H C(n=6)$ were subjected to CD3/CD31 and CD3/CXCR4 double immunofluorescence staining.

Results: The percentage of circulating Tang cells was not different between the whole SSc patient cohort (median 29.9, interquartile range (IQR) 22.3-36.2) and HC (median 25.2, IQR 23.3-33.5). Subgroup analysis revealed that Tang cells were significantly increased in SSc patients with digital ulcers (DU) (median 35.5, IQR 32.2-42.5) compared either with SSc patients without DU (median 23.3, IQR 18.5-26.6) or with HC ( $p<0.0001$ for both). Furthermore, Tang cell percentage was significantly higher in SSc patients with "late" nailfold videocapillaroscopy (NVC) pattern (median 34.9, IQR 25.0-42.0) than in those with "early"/"active" NVC patterns (median 26.5, IQR 20.4-32.9) and in HC ( $p=0.01$ and $p=0.04$, respectively). No difference in circulating Tang cell counts was found when comparing either SSc patients without DU or patients with "early"/"active" NVC patterns and HC. In SSc peripheral blood, the percentage of Tang cells was inversely correlated to the levels of SDF- $1 \alpha$ (Spearman's rho $=-0.59, p<0.0001$ ). Immunohistologic assessment of SSc skin sections revealed the presence of Tang cells in perivascular inflammatory infiltrates.

Conclusions: Our findings demonstrate for the first time that Tang cells are expanded in patients with SSc displaying most severe peripheral vascular complications. Such an expansion may be an ineffective attempt to compensate the need for increased angiogenesis and endothelial progenitor cell function. In SSc, Tang cells might represent a potentially useful biomarker reflecting peripheral vascular damage severity. Further studies are required to clarify the function of Tang cells and investigate the mechanisms responsible for their change in SSc.

Disclosure of Interest: None declared DOI: 10.1136/annrheumdis-2017-eular.3017 